\title{
Macroflora y macrofauna asociada a las raíces sumergidas de Rhizophora mangle (Rhizophoraceae), en la laguna Tampamachoco, Veracruz, México
}

\author{
Macroflora and macrofauna associated with sumerged roots of Rhizophora \\ mangle (Rhizophoraceae), in the Tampamachoco lagoon, Veracruz, Mexico
}

Lucas M, Edith ${ }^{1}$ Biol. Mar; De la Cruz-Francisco, Vicencio ${ }^{1 *}$ M.Sc.

${ }^{1}$ Universidad Veracruzana, Carrera de Biología Marina, Facultad de Ciencias Biológicas y Agropecuarias. Tuxpan, Veracruz, México.

\section{KEYWORDS:}

Amphibalanus; Crassostrea rhizophorae; Ischadium recurvum; macroalgae; red mangrove.

\section{PALABRAS CLAVE:}

Amphibalanus; Crassostrea rhizophorae; Ischadium recurvum; Mangle rojo; macroalgas.

\section{ABSTRACT}

In the Tampamachoco lagoon, Veracruz, the diversity, abundance and similarity of the floristic and faunistic composition associated to $R$. mangle roots were determined; four sampling sites were distributed in the northern, central and southern portions of the lagoon, and 10 mangrove roots were searched for each site for collection, identification and quantification of the organisms. With the quantitative information, species richness, Simpson dominance, fairness and true diversity were estimated, and similarities/dissimilarities were determined between the sampling areas by sorting and ordering analysis. Ten species of algae, 26 invertebrate species and one vertebrate species were identified, totaling 37 species. A total of 10260 macrofaunal organisms were quantified, with an average abundance of $85.5 \pm 71.2$ ind/root being estimated. The most abundant species were Amphibalanus sp., Ischadium recurvum and Crassostrea rhizophorae. The remarkable abundance in the northern zone was significantly different from the central and southern zones. In general, the macrofaunistic community was equitable, and presented true diversity values from 2.8 to 3.7 effective species. The composition and abundance of macrofauna were similar in the three sampling zones. The submerged roots of $R$. mangle play a key role in the Tampamachoco lagoon by providing habitats for various aquatic organisms.

\section{RESUMEN}

En la laguna Tampamachoco, Veracruz se determinó la diversidad, abundancia y similitud de la composición florística y faunística asociada a raíces de $R$. mangle. En la porción norte, centro y sur de la laguna se distribuyeron cuatro sitios de muestreo, por cada sitio se revisaron 10 raíces de mangle para la recolecta, identificación y cuantificación de los organismos. Con la información cuantitativa se estimó la riqueza de especies, dominancia de Simpson, equitatividad y diversidad verdadera, así también se determinaron similitudes/ disimilitudes entre las zonas de muestreo mediante análisis de clasificación y ordenación. Se identificaron diez especies de algas, 26 especies de invertebrados y una especie de vertebrado, que suman un total de 37 especies. Se cuantificó un total de 10260 organismos macrofaunales, estimándose una abundancia promedio de $85.5 \pm 71.2 \mathrm{ind} / \mathrm{raíz}$. Las especies más abundantes fueron Amphibalanus sp., Ischadium recurvum y Crassostrea rhizophorae. La notable abundancia en la zona norte fue significativamente diferente con las zonas centro y sur. En general la comunidad macrofaunística fue equitativa y presentó valores de diversidad verdadera de 2.8 a 3.7 especies efectivas. La composición y abundancia de la macrofauna fue similar en las tres zonas de muestreo. Las raíces sumergidas de $R$. mangle si desempeñan un papel clave en la laguna de Tampamachoco al proporcionar hábitats para diversos organismos acuáticos.
Recibido: 21-08-2017;

Aceptado: 28-12-2017.

Correspondencia autor:

delacruzf17@hotmail.com 


\section{INTRODUCCIÓN}

El mangle rojo (Rhizophora mangle L.) es una especie arbórea que habita en las zonas inundables de estuarios y lagunas costeras (Agraz-Hernández et al., 2006), se caracteriza por poseer raíces muy prominentes que se extienden hacia el sustrato en forma de zancos diseñados para habitar en áreas inundables (Pizarro et al., 2004). Una de las peculiaridades más importantes de las raíces sumergidas de $R$. mangle es la de proveer un sustrato firme a una gran cantidad de organismos acuáticos como algas (Barrios et al., 2003; González et al., 2008) e invertebrados sésiles y sedentarios, los cuales pueden ser de origen marino y/o propiamente lagunar (Reyes y Campos, 1992; Quirós-Rodríguez y Arias, 2013; Ruiz y López-Portillo, 2014).

Colombia y Venezuela son los principales países latinos que han generado un amplio conocimiento sobre las comunidades de algas e invertebrados que se asocian a las raíces de $R$. mangle, principalmente han realizado inventarios florísticos (Barrios et al., 2003; López et al., 2009) así como estudios detallados de la diversidad, abundancia y distribución de diversos grupos faunísticos: esponjas, briozoos, cnidarios, anélidos, moluscos, crustáceos y ascidias (Orihuela y Díaz, 1991; Reyes y Campos, 1992; Márquez y Jiménez, 2002; Vilardy y Polanía, 2002; Medina et al., 2005; Márquez-Rojas et al., 2006; García y Palacio, 2008; Prüsmann y Palacio, 2008; Quiceno y Palacio, 2008; Romero-Murillo y Polanía, 2008; Cedeño et al., 2010; Guerra-Castro et al., 2011; Quirós-Rodríguez y Arias, 2013; Acosta et al., 2014; Quirós-Rodríguez et al., 2017).

En México, a pesar que los manglares se distribuyen en el Pacifico mexicano, Golfo de México y mar Caribe (López-Portillo y Ezcurra, 2002), existen contadas contribuciones sobre comunidades acuáticas asociadas a raíces de $R$. mangle. Para las costas del Pacifico mexicano se han realizado estudios específicos sobre comunidades de cirrípedos (Salgado y Hendrickx, 2002a), cangrejos braquiuros (Salgado y Hendrickx, 2002b) e isópodos (García-Guerrero y Hendrickx, 2003). En el Caribe mexicano las contribuciones científicas tratan sobre algas (Collado-Vides et al., 1995) e invertebrados (Inclán-Rivadeneyra, 1989). Mientras para las costas mexicanas del Golfo de México, solamente existe un trabajo realizado por Ruiz y López-Portillo (2014) quienes determinaron variaciones espaciales y temporales de macroinvertebrados en la laguna La Mancha, Veracruz. Aunado a este panorama, es necesario seguir generando conocimiento al respecto. Por ello, el presente estudio se realizó en la laguna Tampamachoco, Veracruz para caracterizar los ensamblajes de macroflora y macrofauna que se asocian a las raíces sumergidas de $R$. mangle y para determinar similitudes de la comunidad epibionte entre zonas de manglar ubicados cerca de asentamientos humanos y con actividad pesquera, marítima e industrial (Calva y Torres-Alvarado, 2010; De la Cruz-Francisco, 2012) y con zonas sujetas a menor perturbación antrópica.

\section{MATERIALES Y MÉTODOS}

Área de estudio. La laguna de Tampamachoco se localiza en el municipio de Tuxpan, Veracruz, entre las coordenadas $21^{\circ} 02^{\prime} 10^{\prime \prime} \mathrm{N}, 97^{\circ} 22^{\prime} 50^{\prime \prime} \mathrm{W}$ y $20^{\circ}$ $58^{\prime} 40^{\prime \prime} \mathrm{N}, 97^{\circ} 20^{\prime} 29^{\prime \prime} \mathrm{W}$ (Fig. 1). Es un sistema lagunar somero con una profundidad promedio de 1 $\mathrm{m}$, de turbiedad elevada y con transparencia media de $0.30 \mathrm{~cm}$ (Contreras, 1983). Presenta un rango de salinidad 20 a 40 ups, lo que significa que la laguna es de características meso-polihalinas, mientras la temperatura superficial fluctúa entre $25-30^{\circ} \mathrm{C}$ (Lara et al., 2011). La salinidad se incrementa en primavera y decrece en otoño, mientras la temperatura es mayor en verano y disminuye en invierno (López-Ortega et al., 2012). La laguna presenta una longitud y anchura aproximada de $10.6 \mathrm{~km}$ y $2.7 \mathrm{~km}$ respectivamente, y un área de $15 \mathrm{~m}^{2}$. Está separada del mar por una barrera arenosa conocida como "Barra Galindo". Al norte de la laguna, existen dos canales de navegación paralelos a la costa, "Canal antiguo" y "Canal nuevo", este último utilizado actualmente para la navegación y comunica con la Laguna de Tamiahua. Recibe agua del mar por la entrada artificial del estero "Galindo". Hacia la porción sur, la laguna se comunica con el río Tuxpan a través el estero Tampamachoco (Ocaña y Sánchez, 2003). Al centro de la laguna existen isletas orientadas paralelo a la costa que conducen al canal de navegación. En los márgenes de la laguna y en las isletas se desarrolla $R$. mangle. Al extremo sur de la laguna existen asentamientos humanos y hacia el este propiedades ejidales e industriales.

Diseño de muestreo. Los muestreos se realizaron durante las mareas más bajas del mes noviembre 2014 y marzo 2015. Para tal fin se establecieron tres zonas de estudio, la zona norte $(\mathrm{N})$ se estableció al norte del canal de navegación, la zona centro $(\mathrm{C})$ ubicada en las isletas, y la zona sur (S) ubicado a $700 \mathrm{~m}$ de las cabañas y a $1 \mathrm{~km}$ de la localidad "La Mata de Tampamachoco". En cada zona se distribuyeron cuatro sitios de muestreos y por cada sitio se revisaron diez raíces de $R$. mangle las cuales fueron elegidas al azar (Figura 1).

A cada raíz de $R$. mangle se realizaron raspados de la corteza con una espátula para la obtención de todos los organismos posibles, este procedimiento se realizó in situ, si bien, esto implicó más tiempo de trabajo, pero se evitó cortar las raíces del mangle rojo. El material biológico recolectado se almacenó ̧̧冖 


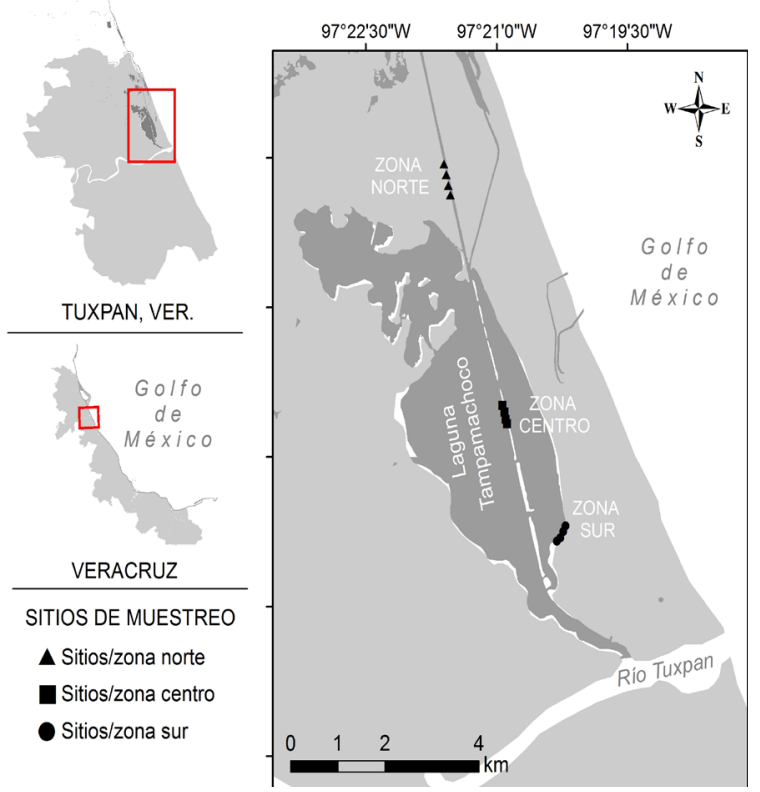

Figura 1. Localización geográfica de la laguna Tampamachoco, Veracruz, México. Se muestran las zonas y sitios de muestreo.

bolsas de polietileno con el fin de evitar la pérdida de organismos. Varios grupos biológicos como crustáceos, gasterópodos y bivalvos se identificaron en campo con base en sus atributos morfológicos y de coloración. Los organismos dudosos se fijaron en formol al $5 \%$ con agua del medio, y posteriormente fueron fijados en alcohol al $70 \%$ y depositados en la Colección Biológica de la Facultad de Biología, Universidad Veracruzana, Campus Tuxpan. Los organismos recolectados fueron identificados utilizando claves taxonómicas y guías de identificación para algas (Littler y Littler, 2000), esponjas (Weerdt et al., 1991), cnidarios (Mills et al., 2007), moluscos (García-Cubas y Reguero, 2004, 2007), anfípodos (Heard, 1982; Díaz et al., 2005), tanaidáceos (Heard et al., 1982), isópodos (Naylor, 1972), balanos (Celis et al., 2007), decápodos (Abele y Kim, 1986; Pérez-Mozqueda et al., 2014) y peces (Montes de Oca, 2010).

La eficiencia de los muestreos se evaluó utilizando el estimador no paramétrico Chao 2, para ello se calculó la representatividad del muestreo (\%) dividiendo el valor final de la riqueza observada (multiplicado por 100) entre el valor final de la riqueza esperada del estimador no paramétrico. Así mismo, se evaluó el desempeño del estimador considerando el nivel de sesgo y exactitud con base en las formulas propuestas por Walther y Moore (2005):; , en ambas fórmulas se obtienen valores que varían entre -1 y 1 , cuando los valores son cercanos a cero indica menos sesgo o mayor exactitud respectivamente. Los valores de la riqueza observada y estimada se obtuvieron con el programa EstimateS (usando 100 aleatorizaciones) versión 9 (Colwell, 2013) y se graficaron para representar las curvas acumulativas de especies observadas y estimadas. También se utilizó el modelo asintótico de Clench para predecir el número de especies existentes en cada sitio de estudio, así también para evaluar la calidad del inventario y estimar el esfuerzo de muestreo realizado, todo este proceso se realizó con base en el trabajo de Jiménez-Valverde y Hortal (2003).

Análisis cuantitativo: Únicamente la macrofauna móvil mayor a $1 \mathrm{~mm}$ fue contabilizada en el estudio, principalmente se estimó la abundancia numérica (número de individuos por raíz) de anélidos, moluscos, crustáceos y peces, por lo que en este análisis se excluyeron organismos sésiles de características coloniales e incrustantes como: algas, esponjas, briozoarios y cnidarios. En cada sitio de muestreo se revisaron diez raíces sumergidas de $R$. mangle para la identificación y conteo de especies epibiontes vivas, con esta información se estimaron índices basados en la abundancia: riqueza específica, diversidad de Shannon, equitatividad, dominancia de Simpson (D) y diversidad verdadera de orden 1 (1D), cada atributo comunitario fue estimado a nivel de muestra (raíz) y fue promediado a nivel de sitio de muestreo. La diversidad verdadera (1D) se obtuvo calculando el exponente de la diversidad de Shannon: $1 D=\exp H^{\prime}$, este índice expresa el número efectivo de especies que tendría una comunidad integrada por especies igualmente abundantes, dicho índice tiene la ventaja de realizar comparaciones de la diversidad verdadera de dos o más comunidades (Moreno et al., 2011). Para cada atributo comunitario se determinaron diferencias significativas entre los sitios de muestreo de cada zona estudiada mediante análisis de varianza (ANDEVA), para ello se utilizó el programa Statistica 7.1 (StatSoft, 2005).

Se utilizó el índice de Bray-Curtis para determinar similitudes/disimilitudes entre las zonas de muestreo con base en la composición y abundancia de la macrofuana (excluyendo organismos sésiles), a partir de la matriz de similitud se generó un dendrograma a fin de mostrar el grado de semejanza entre los sitios de muestreo. Como análisis complementario se utilizó el método de ordenación multidimensional no métrico (nMDS), basándose de la matriz de similitud de Bray-Curtis para representar en dos dimensiones las proximidades de los sitios de muestreo; también se realizó el análisis de similitud porcentual (SIMPER) para determinar cuáles especies contribuyen en la diferenciación/similitud de los grupos formados, y para averiguar diferencias significativas se utilizó la prueba de similitud ANOSIM, dichos análisis multivariantes se realizaron con el programa Primer Versión 7 (Clarke y Gorley, 2015). 


\section{RESULTADOS}

Un total de 120 raíces de $R$. mangle fueron revisadas, identificándose 10 especies de algas, representadas en dos reinos (Chromista y Plantae) y tres phyla: Ochrophyta (1 sp.), Rhodophyta (6 spp.) y Clorophyta (3 spp.). Así mismo, se identificaron los siguientes phyla del reino Animal: Porifera (2 especies), Cnidaria (2spp), Annelida (1 sp.), Arthropoda (12 spp.), Mollusca (8 spp.), Bryozoa (1 sp.) y Chordata (1 sp.), que suman un total de 27 especies faunísticas (Tabla 1).

En la porción norte se registraron 15 especies faunísticas (no se hallaron algas), con base en el estimador no paramétrico Chao 2 , la representatividad del muestreo fue buena $(91,1 \%)$ dado que la predicción fue 16,46 especies, mientras la predicción de Clench fue de 15,2 especies ( $a=5.414125 ; b=0.356004)$ lo que reafirma un esfuerzo de muestreo eficiente $(98,6 \%)$ para esta zona. Estos resultados se reflejan en la curva acumulativa de especies observadas y esperadas (Figura 2a).

Para la zona centro, se registró mayor cantidad de riqueza encontrándose 10 especies de algas y 19 de invertebrados marinos para un total de 29 especies, el estimador Chao 2 presentó un mal desempeño dado que sobrestimó la riqueza de este lugar con una predicción de 58 especies, en cambio el modelo de Clench estimó 34,2 especies, esto equivale a un $84,7 \%$ de representatividad en el muestreo, para registrar el $95 \%$ de la riqueza de especies es necesario aumentar el esfuerzo de muestreo a 10 muestras más, en consecuencia las curvas acumulativas de especies no alcanzaron la asíntota (Figura 2b).

En la porción sur, tampoco se observaron algas, solamente se registraron 17 especies faunísticas, esta cifra se aproxima a la predicción del estimador Chao 2 que fue de 17,33 especies, por lo tanto la representatividad del muestreo fue buena $(98,1 \%)$, el modelo de Clench reafirma la eficiencia en el muestreo con la predicción de 18 especies en esta zona, lo que equivale a un $94,3 \%$ de representatividad, para registrar al menos el $95 \%$ de la fauna existente en este lugar se requiere aumentar el esfuerzo de muestreo a 10 muestras más. Para esta zona, las curvas acumulativas de especies presentaron tendencia a la asíntota (Figura 2c).

Análisis cuantitativo. Se contabilizó un total de 10260 organismos faunísticos en las raíces de $R$. mangle, identificándose 22 especies, la abundancia promedio fue de $85.5 \pm 71.2$ ind/raíz. Las especies
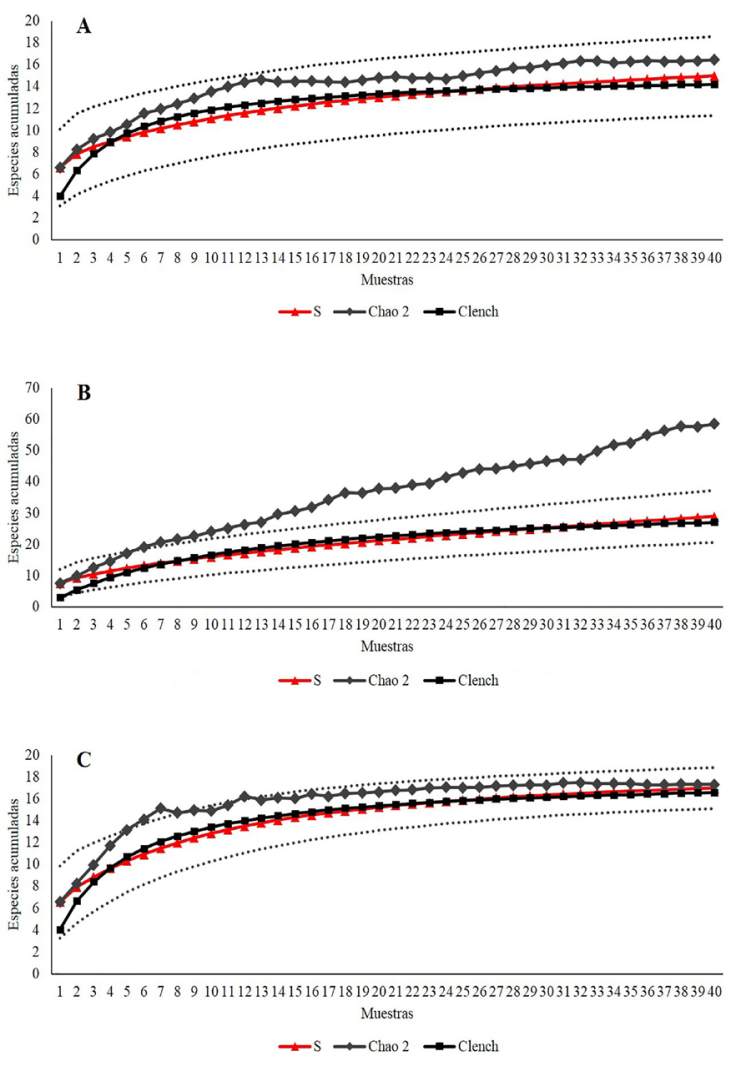

Figura 2. Número de especies observadas (S) y estimadas (Chao 1 y Clench) para las zonas A) norte, B) centro y C) sur correspondiente a la macroflora y macrofauna asociada a las raíces de $R$. mangle, laguna Tampamachoco, Veracruz. Las líneas punteadas indican el intervalo de confianza (superior $\mathrm{e}$ inferior) a $95 \%$ de las especies observadas.

más abundantes en el estudio fueron Amphibalanus sp., Ischadium recurvum y Crassostrea rhizophorae (Fig. 3), para las restantes especies se registraron abundancias menores de 10 organismos. Por otra parte, en la zona norte se registró mayor abundancia promedio con $141 \pm 90.3$ organismos, mientras en la zona sur la abundancia promedio fue menor con $48.1 \pm 36.2$ organismos (Tabla 2). Las especies más representativas en la zona norte fueron $I$. recurvum

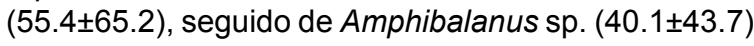
y Mytilopsis leucophaeata $(26.7 \pm 27.7)$. Para la zona centro las más abundantes fueron Amphibalanus sp.

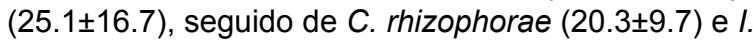
recurvum (13.2 \pm 12.4 ), mientras que para la zona sur las especies con mayor número de individuos fueron $C$.

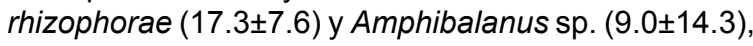
las restantes especies manifestaron abundancias promedio $<5$ individuos (Tabla 2 ). 
Tabla 1. Lista florística y faunística registrada en las raíces sumergidas de Rhizophora mangle, laguna Tampamachoco, Veracruz, México. Zonas de muestreo, Norte (N), Centro (C), Sur (S).

\begin{tabular}{|c|c|c|c|c|}
\hline Categorías & Especie & $\mathbf{N}$ & C & $\mathbf{S}$ \\
\hline \multicolumn{5}{|l|}{$\begin{array}{l}\text { Reino Chromista } \\
\text { Phylum Ochrophyta } \\
\text { Clase Phaeophyceae }\end{array}$} \\
\hline $\begin{array}{l}\text { Acinetosporaceae } \\
\text { Reino Plantae } \\
\text { Phylum Rhodophyta } \\
\text { Clase Florideophyceae }\end{array}$ & Hincksia mitchelliae (Harvey) P.C.Silva & & $\boldsymbol{\Delta}$ & \\
\hline Ceramiaceae & Ceramium cruciatum Collins \& Hervey & & $\boldsymbol{\Delta}$ & \\
\hline Cystocloniaceae & Hypnea spinella (C.Agardh) Kützing & & $\overline{\boldsymbol{\Delta}}$ & \\
\hline Gelidiellaceae & Gelidiella acerosa (Forsskål) Feld́mann \& Hamel & & $\Delta$ & \\
\hline Gracilariaceae & $\begin{array}{c}\text { Gracilaria mammillaris (Montagne) M.Howe } \\
\text { Gracilaria blodgettii Harvey }\end{array}$ & & $\overline{\mathbf{\Delta}}$ & \\
\hline $\begin{array}{l}\text { Rhodomelaceae } \\
\text { Phylum Chlorophyta } \\
\text { Clase Ulvophyceae }\end{array}$ & Laurencia obtusa (Hudson) J.V.Lamouroux & & $\boldsymbol{\Delta}$ & \\
\hline Ulvaceae & $\begin{array}{c}\text { Enteromorpha flexuosa (Wulfen) J.Agardh } \\
\text { Ulva fasciata Delile }\end{array}$ & & $\dot{\Delta}$ & \\
\hline $\begin{array}{l}\text { Bryopsidaceae } \\
\text { Reino Animalia } \\
\text { Phylum Porifera } \\
\text { Clase Demospongiae }\end{array}$ & Bryopsis sp. & & $\overline{\boldsymbol{\Lambda}}$ & \\
\hline Microcionidae & Clatrhia sp. & & $\boldsymbol{\Delta}$ & \\
\hline \multicolumn{5}{|l|}{$\begin{array}{l}\text { Phylum Cnidaria } \\
\text { Clase Hydrozoa }\end{array}$} \\
\hline Campanulariidae & $\begin{array}{c}\text { Obelia bidentata Clark, } 1875 \\
\text { Obelia longissima (Pallas, } 1766 \text { ) }\end{array}$ & & & $\bullet$ \\
\hline \multicolumn{5}{|l|}{$\begin{array}{l}\text { Phylum Annelida } \\
\text { Clase Polychaeta }\end{array}$} \\
\hline \multicolumn{4}{|l|}{$\begin{array}{l}\text { Phylum Arthropoda } \\
\text { Clase Malacostraca }\end{array}$} & $\bullet$ \\
\hline Grapsidae & $\begin{array}{l}\text { Goniopsis cruentata (Latreille, 1803) } \\
\text { Grapsus grapsus (Linnaeus, 1758) }\end{array}$ & . & $\boldsymbol{\Delta}$ & $\bullet$ \\
\hline Sersamidae & Aratus pisonii $(\mathrm{H}$. Milne Edwards, 1837) & & $\boldsymbol{\Delta}$ & $\bullet$ \\
\hline Panopeidae & Eurytium limosum (Say, 1818) & - & & \\
\hline Ocypodidae & Minuca rapax (Smith, 1870) & - & & $\bullet$ \\
\hline Porcellanidae & Petrolisthes armatus (Gibbes, 1850) & & $\Delta$ & \\
\hline Sphaeromatidae & Sphaeroma terebrans Bate, 1866 & - & $\overline{\boldsymbol{\Delta}}$ & $\bullet$ \\
\hline Caprellidae & Caprella equilibra Say, 1818 & . & $\Delta$ & $\bullet$ \\
\hline Gammaridae & Gammaridae sp. & - & $\Delta$ & • \\
\hline $\begin{array}{l}\text { Leptocheliidae } \\
\text { Clase Hexanauplia }\end{array}$ & Leptocheliidae sp. & $\overline{-}$ & $\overline{\boldsymbol{\Delta}}$ & $\bullet$ \\
\hline Balanidae & $\begin{array}{l}\text { Amphibalanus sp. } \\
\text { Megabalanus coccopoma (Darwin, 1854) }\end{array}$ & ! & $\hat{\Delta}$ & $\bullet$ \\
\hline \multicolumn{5}{|l|}{$\begin{array}{l}\text { Phylum Mollusca } \\
\text { Clase Gastropoda }\end{array}$} \\
\hline Littorinidae & $\begin{array}{l}\text { Littoraria angulifera (Lamarck, 1822) } \\
\text { Littoraria nebulosa (Lamarck, 1822) }\end{array}$ & & $\dot{\Delta}$ & $\bullet$ \\
\hline $\begin{array}{l}\text { Neritidae } \\
\text { Muricidae }\end{array}$ & $\begin{array}{l}\text { Neritina usnea (Röding, 1798) } \\
\text { Stramonita haemastoma (Linnaeus, 1767) }\end{array}$ & - & $\Delta$ & \\
\hline \multicolumn{5}{|l|}{ Clase Bivalvia } \\
\hline $\begin{array}{l}\text { Ostreidae } \\
\text { Pteriidae }\end{array}$ & $\begin{array}{l}\text { Crassostrea rhizophorae (Gullding, 1828) } \\
\text { Isognomon alatus (Gmelin, 1791) }\end{array}$ & - & $\hat{\Delta}$ & $\bullet$ \\
\hline Mytilidae & Ischadium recurvum (Rafinesque, 1820) & ! & $\overline{\boldsymbol{\Delta}}$ & $\bullet$ \\
\hline Dreissenidae & Mytilopsis leucophaeata (Conrad, 1831) & . & & \\
\hline $\begin{array}{l}\text { Phylum Bryozoa } \\
\text { Phylum Chordata } \\
\text { Clase Actynopterigii }\end{array}$ & & $\overline{-}$ & $\boldsymbol{\Delta}$ & $\bullet$ \\
\hline \multirow[t]{2}{*}{ Blenniidae } & Lupinoblennius nicholsi (Tavolga, 1954) & - & & \\
\hline & Total de especies & 15 & 29 & 17 \\
\hline
\end{tabular}




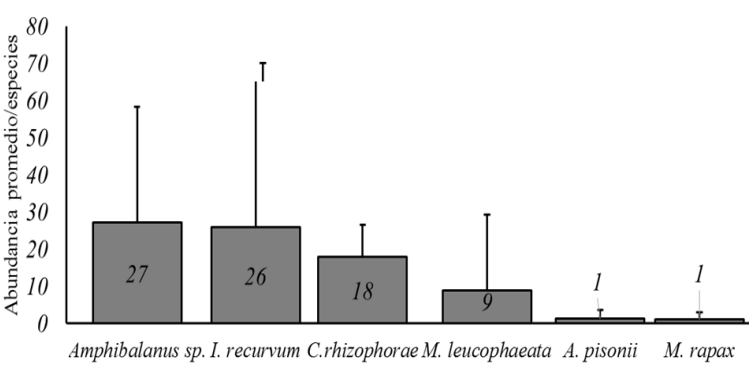

Figura 3. Especies faunísticas más abundantes registradas en raíces de Rhizophora mangle, laguna Tampamachoco, Veracruz, México.

La riqueza promedio no varió considerablemente entre los sitios de muestreo registrándose entre cinco y seis especies, sin embargo el análisis de varianza indicó diferencias significativas $(F=9.50 ; P=0.0061)$, siendo la zona centro diferente estadísticamente de las zonas norte y sur (Figura 4A). En cuanto a la abundancia promedio, evidentemente hay diferencias estadísticas $(F=17.45 ; P=0.0008)$ por la notable abundancia que manifestaron los sitios de la zona norte (Figura 4B). Respecto a los índices ecológicos, en todos los sitios de muestreo se estimaron valores bajos de dominancia, lo que significa que se registraron valores altos de equitatividad (Figura 5A), el análisis estadístico indicó ausencia de diferencias significativas en la dominancia $(F=1.58 ; P=0.257)$, pero si discrepancias significativas en la equitatividad ( $F=5.79 ; P=0.0242)$. Mientras la diversidad verdadera fue parecida en todos los sitios de muestreo, no obstante los sitios N3 y N4 presentaron menor número de especies efectivas ( $\left.{ }^{1} D=2.8\right)$, mientras el máximo valor en este atributo fue de 3.7 especies efectivas $\left({ }^{1} \mathrm{D}\right)$ para el sitio S4 (Figura $5 B$ ).


Figura 4. Valores promedio de riqueza (A) y abundancia (B) correspondiente a la fauna registrada en raíces de $R$. mangle, laguna Tampamachoco, Veracruz.

Tabla 2. Abundancia promedio de las especies faunísticas registradas en raíces de $R$. mangle, laguna Tampamachoco, Veracruz, México.

\begin{tabular}{ccccc}
\hline & Especies & Norte & Centro & Sur \\
\hline Phylum Annelida & Nereididae sp. & $0.1 \pm 0.3$ & $0.1 \pm 0.3$ & $0.1 \pm 0.3$ \\
Phylum Arthropoda & G. cruentata & - & $0.1 \pm 0.2$ & $0.1 \pm 0.3$ \\
& G. grapsus & $1.7 \pm 2.5$ & - & - \\
& A. pisonii & - & $3.8 \pm 2.8$ & $0.1 \pm 0.3$ \\
& E. limosum & $<.1$ & - & - \\
& M. rapax & $<0.1$ & - & $3.1 \pm 2.5$ \\
& P. armatus & - & - & 1.0 \\
& S. terebrans & 1.0 & 1.0 & 0.2 \\
& C. equilibra & $0.1 \pm 0.3$ & $0.1 \pm 0.3$ & 0.2 \\
& Gammaridae sp. & $0.1 \pm 0.3$ & $0.2 \pm 0.4$ & $16.3 \pm 21.7$ \\
& Amphibalanus sp & $40.1 \pm 43.7$ & $25.1 \pm 16.7$ & $<0.1$ \\
& M. coccopoma & - & $0.1 \pm 0.2$ & $0.2 \pm 0.4$ \\
Phylum Mollusca & Leptocheliidae sp. & $0.2 \pm 0.4$ & $0.2 \pm 0.4$ & $0.1 \pm 0.3$ \\
& L. angulifera & - & $0.5 \pm 0.8$ & $<0.1$ \\
& L. nebulosa & - & $<0.1$ & - \\
& N. usnea & $0.1 \pm 0.3$ & - & $17.3 \pm 7.6$ \\
& S. haemastoma & - & $<0.1$ & $0.7 \pm 3.0$ \\
& C.rhizophorae & $16.2 \pm 8.3$ & $20.3 \pm 9.7$ & $9.0 \pm 14.3$ \\
& I. alatus & - & $2.2 \pm 2.8$ & - \\
I. recurvum & $55.4 \pm 65.2$ & $13.2 \pm 12.4$ & - \\
\hline & M. leucophaeata & $26.7 \pm 27.7$ & - & $48.1 \pm 36.2$
\end{tabular}






De acuerdo al análisis de similitud de Bray-Curtis, la comunidad faunística asociada a las raíces de $R$. mangle es semejante en composición y abundancia de especies (Fig. 6A); en el análisis nDMS se muestra claramente la separación de los sitios de la zona norte con respecto a los sitios de la zona sur y centro, inclusive el sitio S3 presento mayor similitud con los sitios de la zona centro (Fig. 6B), no obstante las disimilitudes existentes entre las zonas son significativas de acuerdo al ANOSIM (R global= 0.964; $P=0.001$ ). Con base en el análisis SIMPER, tres especies contribuyeron en la similitud de los sitios de la zona norte y centro, y cuatro especies favorecieron la similitud de los sitios de la zona sur (Tabla 3). De acuerdo al análisis ANOSIM por pares, la zona norte y centro son significativamente diferentes

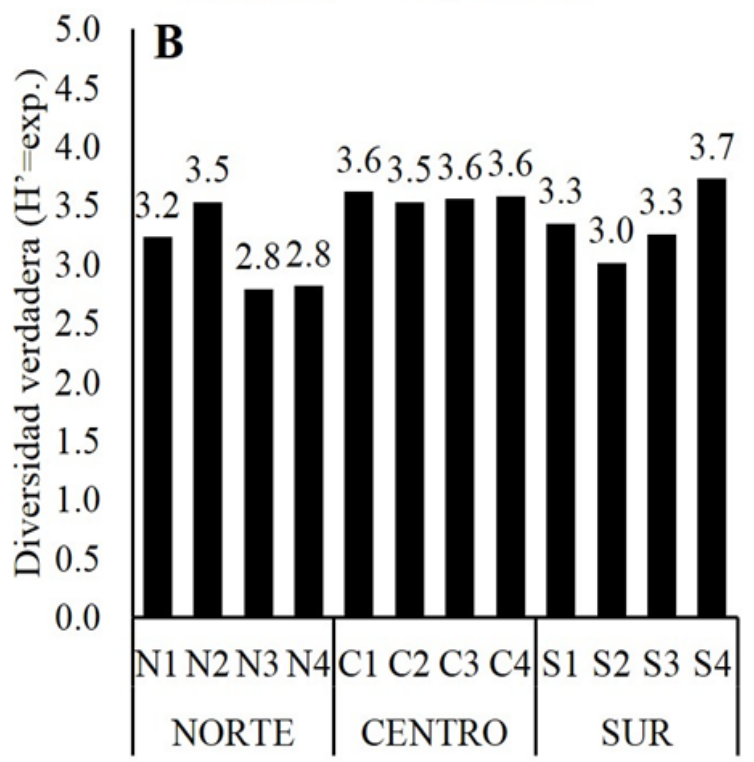

Figura 5. Valores promedio de la equitatividad, dominancia (A) y diversidad verdadera (B) correspondiente a la fauna registrada en raíces de $R$. mangle, laguna Tampamachoco, Veracruz.

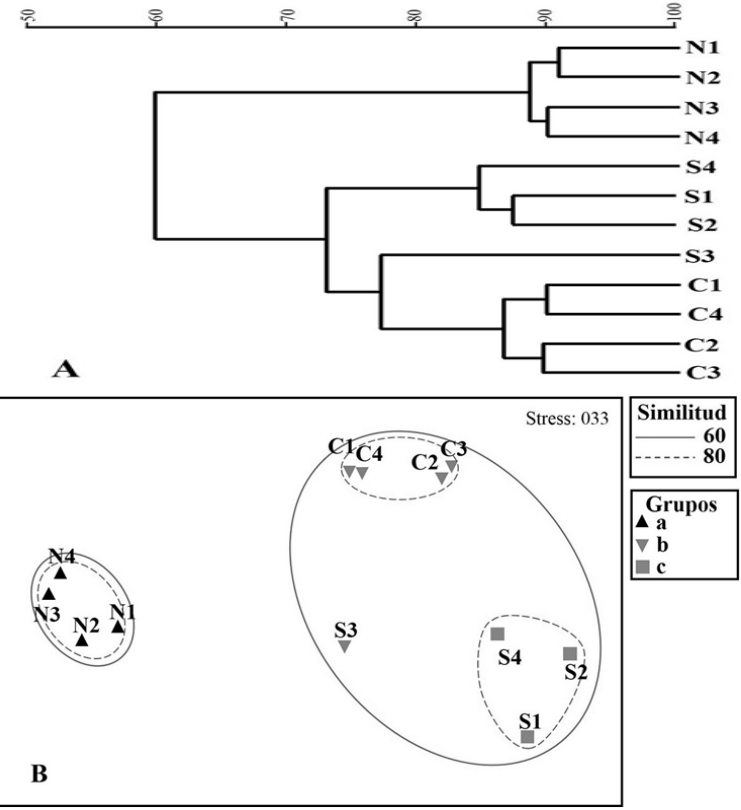

Figura 6. Análisis de similitud de Bray-Curtis y análisis nMDS aplicado a la fauna contabilizada en las raíces de $R$. mangle, laguna de Tampamachoco, Veracruz, México. Zonas de muestreo, Norte (N), Centro (C), Sur (S).

Tabla 3. Valores de similitud porcentual (SIMPER) de las especies macrofaunísticas que contribuyeron en la semejanza de las zonas de muestreo, laguna Tampamachoco, Veracruz, México.

\begin{tabular}{ccccc}
\hline Zonas & Especies & Similitud promedio & \% contribución & \% acumulado \\
\hline \multirow{2}{*}{ Norte } & I. recurvum & $24.90 \pm 15.34$ & 27.85 & 27.85 \\
$(89.42 \%)$ & Amphibalanus sp. & $20.43 \pm 6.97$ & 22.85 & 50.70 \\
& M. leucophaeata & $16.75 \pm 4.97$ & 20.67 & 79.24 \\
Centro & Amphibalanus sp. & $23.78 \pm 12.03$ & 28.49 & 28.49 \\
$(83.48 \%)$ & C. rhizophorae & $20.95 \pm 19.38$ & 25.10 & 53.59 \\
& I. recurvum & $16.63 \pm 8.14$ & 19.92 & 73.50 \\
Sur (85.67\%) & C. rhizophorae & $26.68 \pm 35.58$ & 31.14 & 31.14 \\
& Amphibalanus sp. & $16.22 \pm 432.57$ & 18.93 & 50.07 \\
& I. recurvum & $14.26 \pm 11.66$ & 16.65 & 66.72 \\
\end{tabular}


Tabla 4. Especies macrofaunísticas que contribuyeron en la disimilitud entre la zona de muestreo: norte + centro; norte + sur; centro + sur. $\mathrm{AP}=\mathrm{Abundancia}$ promedio; $\mathrm{CBT} \%=$ Contribución porcentual de cada especie; $\mathrm{ACM} \%=$ Porcentaje acumulativo de cada especie.

\begin{tabular}{|c|c|c|c|c|}
\hline Disimilitud norte + centro $=36.27 \%$ & AP(norte) & AP(centro) & CTB $\%$ & ACM\% \\
\hline M. leucophaeata & 16.26 & 0 & 30.37 & 30.37 \\
\hline l. recurvum & 23.34 & 11.79 & 21.53 & 51.89 \\
\hline A. pisoni & 0 & 4.82 & 9.02 & 60.91 \\
\hline Amphibalanus sp. & 19.74 & 16.64 & 7.88 & 68.79 \\
\hline G. grapsus & 4.08 & 0 & 7.64 & 76.43 \\
\hline Disimilitud norte + sur $=46.23 \%$ & AP(norte) & $\mathrm{AP}$ (sur) & CTB\% & $\mathrm{ACM} \%$ \\
\hline M. leucophaeata & 16.26 & 0 & 26.65 & 26.65 \\
\hline I. recurvum & 23.34 & 7.55 & 25.82 & 52.48 \\
\hline Amphibalanus sp. & 19.74 & 8.82 & 17.85 & 70.33 \\
\hline Disimilitud centro + sur= $26.93 \%$ & $\mathrm{AP}$ (centro) & $\mathrm{AP}$ (sur) & CTB $\%$ & $\mathrm{ACM} \%$ \\
\hline Amphibalanus sp. & 16.64 & 8.82 & 26.32 & 26.32 \\
\hline M. rapax & 1.33 & 5.09 & 14.72 & 41.04 \\
\hline A. pisoni & 4.82 & 0.80 & 14.64 & 55.68 \\
\hline I. recurvum & 11.79 & 7.55 & 14.20 & 69.88 \\
\hline I. alatus & 3.90 & 2.27 & 8.25 & 78.14 \\
\hline
\end{tabular}

$(R=1 ; P=0.008)$, cinco especies contribuyeron en la diferenciación de estos grupos (Tabla 4); así mismo, se determinaron diferencias significativas entre la zona norte y sur $(R=1 ; P=0.029)$, el análisis SIMPER determinó que tres especies contribuyeron en la disimilitud de estas zonas (Tabla 4); de igual manera la disimilitud entre centro y sur fueron significativos $(\mathrm{R}=$ 0.836; $P=0.018$ ), cinco especies contribuyeron en la diferenciación de estos grupos (Tabla 4).

\section{DISCUSIÓN}

Es evidente que las raíces sumergidas de $R$. mangle si cumplen un papel importante en la laguna de Tampamachoco (De la Cruz-Francisco, 2012), dado que fungen como sustratos para una gran variedad de organismos acuáticos. La ficoflora encontrada fue de 10 especies, mismas que han sido reportadas previamente para el sistema lagunar (Vilchis, 20015), no obstante, esta riqueza obtenida es menor dado que en manglares del mar Caribe se han reportado mayor cantidad de especies de algas como en la laguna Nichupte, Quintana Roo (Collado-Vides et al., 1995), en los sistemas lagunares de Venezuela (Orihulea et al., 1991; Barrios et al., 2003; López et al., 2009) y Cuba (Moreira et al., 2003), se argumenta que las condiciones estables en temperatura, salinidad y transparencia del agua han posibilitado una mayor riqueza de algas en estas áreas del Caribe (Collado-Vides et al., 1995; González et al., 2008; López et al., 2009). Así mismo se ha mencionado que en condiciones estresantes como la turbiedad que minimiza la transparencia del agua y/o cambios bruscos de salinidad logran prosperar solamente especies tolerantes al estrés ambiental (Orihulea et al., 1991; Barrios et al., 2003). Contrariamente las características hidrológicas que ocurren en la laguna Tampamachoco no son estables, dado que la temperatura y salinidad varían en el año (De la Lanza-Espino et al., 1998; López-Ortega et al., 2012) y la tasa de sedimentación es alta (Calva y Torres-Alvarado, 2000).
La comunidad faunística observada en raíces de $R$. mangle fue más representativa con 27 especies, cifra que se aproxima a las 28 especies que se reportan para la laguna La Mancha, Veracruz, aunque solamente se comparten ocho especies (Ruiz y López-Portillo, 2014) dado que en este ecosistema lagunar no fueron reportados poríferos y vertebrados. Cabe mencionar que la riqueza faunística de estos sistemas lagunares es menor comparado a los manglares del mar Caribe; Ruiz y López-Portillo (2014) lo atribuyen por la alta turbiedad y variaciones de salinidad y temperatura que presenta la laguna de La Mancha, atributos que son típicos de un sistema de manglar continental, mismos rasgos caracterizan por igual a la laguna de Tampamachoco (De la Lanza-Espino et al., 1998; Calva y Torres-Alvarado, 2000). En cambio los manglares del Caribe reciben mayor influencia del mar y llegan a presentar una mezcla con ambientes cercanos como pastos marinos, formaciones coralinas y rocosas, todo ello favorece que exista mayor riqueza faunística (Orihuela et al., 1991; Reyes y Campos, 1992; Márquez y Jiménez, 2002; Márquez-Rojas et al., 2006; Prüsmann y Palacio, 2008; Cedeño et al., 2010; Guerra-Castro et al., 2011; Quirós-Rodríguez y Arias, 2013; Acosta et al., 2014; Quirós-Rodríguez et al., 2017).

En general, moluscos y artrópodos fueron los más diversos en especies y más abundantes en las raíces de $R$. mangle de la laguna Tampamachoco, mismos resultados también se han citado para los manglares del Caribe Colombiano (Reyes y Campos, 1992; Quiceno y Palacio, 2008; Romero-Murillo y Polanía, 2008; Quirós-Rodríguez y Arias, 2013) y Venezolano (Márquez y Jiménez, 2002; Márquez-Rojas et al., 2006; Cedeño et al., 2010; Acosta et al., 2014), no obstante, con excepción de la laguna La Restinga donde se ha citado a las esponjas como el grupo más diverso y abundante (Orihuela et al., 1991; GuerraCastro et al., 2011).

Básicamente las especies más abundantes en las raíces de $R$. mangle fueron Amphibalanus sp., seguido de I. recurvum, C. rhizophorae y $M$. leucophaeata, este 
ensamblaje faunístico es característico de las raíces sumergidas del mangle rojo dado que igualmente son referidas como las más representativas en la laguna $\mathrm{La}$ Mancha (Veracruz) donde M. leucophaeta la citan como la más numerosa, mientras $C$. rhizophorae e $I$. recurvum las refieren como las de mayor biomasa (Ruiz y LópezPortillo et al., 2014), incluso en un experimento con tubos artificiales las mismas especies resultaron ser las más numerosas para el citado sistema lagunar (Ruiz-Guerrero y López-Portillo, 2017). Al menos C. rhizophorae e I. alatus también son referidas como abundantes en manglares de Venezuela, principalmente en Isla Larga, Bahía de Mochima (Acosta et al., 2014), dado que en la laguna La Restinga (Guerra-Castro et al., 2011) y en el Golfo de Santa Fe (Marqués y Jiménez, 2002) solo citan a C. rhizophorae como abundante en $R$. mangle, también se ha reconocido la abundancia de $C$. rhizophorae en el Caribe Colombiano especialmente en Ciénega Boquilla (Quinceno y Palacio, 2008) y en Bahía de Cispata (Quirós-Rodríguez y Arias, 2013).

En general la macrofauna asociada a las raíces de $R$. mangle fue equitativita en las tres zonas de muestreo, este hecho se atribuye por la notable abundancia $C$. rhizophorae, Amphibalanus sp. e I. recurvum, las cuales son típicas del mangle rojo (Guerra-Castro et al., 2011; Acosta et al., 2014; Ruiz y López-Portillo, 2014), el éxito de estas especies se puede atribuir por la disponibilidad de alimento dado que son de hábitos filtradores y la obtienen de partículas orgánicas suspendidas, elemento que es característico en la laguna de Tampamachoco dada la alta tasa de sedimentación (Calva y Torres-Alvarado, 2000), también se puede atribuir a que se reproducen de manera continua (Montes et al., 2007; Ruiz y López-Portillo, 2014). La equitatividad también se ha reportado en manglares de las bahía de Turbo y EI Uno, Golfo de Urabá (García y Palacio, 2008) y en la Ciénega La Boquilla, Sucre (Quiceno y Palacio, 2008) ambos del Caribe Colombiano.

Con base en los análisis multivariados, la composición y abundancia de la macrofauna asociada a las raíces sumergidas de $R$. mangle fue similar en las tres zonas de muestreo, sin embargo las disimilitudes existentes fueron significativas debido a que la zona norte presentó mayor cantidad de macrofauna, esencialmente por la notable abundancia de I. recurvum, Amphibalanus sp., $M$. leucophaeta y $C$. rhizophorae, en cambio, hacia la zona centro y sur decrecieron las abundancias de estas especies y ausentándose $M$. leucophaeta. Estos cambios en la abundancia sugieren que deben existir condiciones que favorecen mayor asentamiento de macrofauna en la zona norte, esto se puede atribuir a una probable conectividad entre la laguna de Tamiahua hacia la laguna de Tampamachoco a través del canal nuevo, lo cual podría suceder a partir del aporte de larvas, mismas que primeramente deberían de asentarse, fijarse y colonizar la corteza de las raíces del mangle rojo de la zona norte, favoreciendo ensamblajes faunísticos abundantes. Esta zona, también recibe agua de mar por el estero "Galindo" lo que podría favorecer condiciones estables en las características fisicoquímicas (López-Ortega et al., 2012).

Por el contrario, la baja abundancia de macrofauna en la zona centro y sur podría atribuirse a la actividad antrópica, por ejemplo, los pescadores recurren a cortar las raíces de mangle para obtener ejemplares de $C$. rhizophorae para su venta local, otra actividad puede ser el tránsito marítimo, las embarcaciones transitan cerca de los márgenes de mangle que bordean las isletas, lo cual podría alterar las características hidrológicas de estas zonas provocando mayor turbiedad y resuspensión de sedimentos. Además, se presume una mala calidad del agua para estas zonas por aguas residuales debido a la presencia de asentamientos humanos, las cuales se han establecido en la porción sur de la laguna. Así mismo se ha notificado que la laguna recibe aportes de contaminantes orgánicos, inorgánicos y biológicos, los cuales podrían alterar la biota acuática y las condiciones hidrológicas de la laguna de Tampamachoco (Calva y Torres-Alvarado, 2000).

Aunado a lo anterior, se sugiere que en posteriores trabajos se valoren las características hidrológicas de la laguna Tampamachoco a fin de relacionarlo con la abundancia de la macrofauna, la cual también debe ser valorada tanto a nivel espacial y temporal considerando la época de nortes, secas y lluvias (Ruiz y López-Portillo, 2014) con el fin de obtener sólidos argumentos para explicar la dinámica ecológica de la macrofauna que se asocia en las raíces de R. mangle.

En conclusión, las raíces sumergidas de $R$. mangle si constituyen hábitats importantes para el asentamiento de flora y fauna acuática, las cuales deben ser valoradas nuevamente en posteriores estudios a nivel espacial y temporal. La macrofauna fue el grupo más representativo en especies y en abundancia numérica, dicha comunidad fue equitativa en las tres zonas. La zona norte es significativamente diferente a las demás zonas, por la notable abundancia de organismos faunísticos. La comunidad epibionte es propia de un sistema de manglar continental esencialmente caracterizados por la abundancia de especies de bivalvos (I. recurvum y $C$. rhizophorae) y crustáceos cirrípedos (Amphibalanus sp.).

\section{Agradecimientos.}

ARosa Ivette Vásquez Estrada y Josefina Esther Paredes Flores por su apoyo en la recolecta de las muestras, también a Rosa Estela Orduña Medrano por el apoyo en la confirmación taxonómica de las algas. A Jordán Gutiérrez Vivanco y Arturo Valdés Murillo por facilitarnos el material de laboratorio y de microscopia. A los revisores anónimos por sus observaciones y sugerencias. 


\section{REFERENCIAS}

Abele, L.G.; Kim, W. 1986. An ilustrated guide tothe marine decapod crustaceans of Florida. Technical series 8 (1):1-729.

Acosta B, V.; Betancourt T, R.; Prieto A, A. 2014. Estructura comunitaria de bivalvos y gasterópodos en raíces del mangle rojo Rhizophora mangle (Rhizophoraceae) en isla Larga, bahía de Mochima, Venezuela. Revista de Biología Tropical 62 (2):551-565.

Agráz-Hernández, C.M.; Noriega-Trejo, R.; López-Portillo, J.; Flores-Verdugo, F.J.; Jiménez-Zacarías, J.J. 2006. Guía de campo. Identificación de los manglares de México. Universidad Autónoma de Campeche.

Barrios, J.E.; Márquez, B.; Jiménez, M. 2003. Macroalgas asociadas a Rhizophora mangle L. en el golfo de Santa Fe, Estado Sucre, Venezuela. Boletín del Instituto Oceanográfico Universidad de Oriente 42:37-45.

Calva B, L.G.; Torres-Alvarado, R. 2000. Distribución de carbohidratos, carbono y nitrógeno orgánico en sedimentos de tres lagunas costeras del golfo de México. Hidrobiológica 10 (2):101-114.

Cedeño, J.; Jiménez P, M.; Pereda, L.; Allen, T. 2010. Abundancia y riqueza de moluscos y crustáceos asociados a las raíces sumergidas del mangle rojo (Rhizophora mangle) en la laguna de Bocaripo, Sucre, Venezuela. Revista de Biología Tropical 58 (Suppl.3):213-226.

Celis, A.; Rodríguez-Almaráz. G.; Álvarez, F. 2007. Los cirripedios torácicos (Crustacea) de aguas someras de Tamaulipas, México. Revista Mexicana de Biodiversidad 78:325-337.

Clarke, K.R.; Gorley, R.N. 2015. Getting started with PRIMER V7. PRIMER-E. Plymouth, Reino Unido. PRIMER-E Ltd.

Collado-Vides, L.; González-González, J.; Ezcurra, E. 1995. Patrones de distribución ficoflorística en el sistema lagunar de Nichupte, Quintana Roo, México. Acta Botánica Mexicana 31:19-32.

Colwell, R.K. 2013. EstimateS: Statistical estimation of species richness and shared species from samples. Version 9.

Contreras, E. F. 1983. Variaciones en la hidrología y concentraciones de nutrientes del área estuarino-lagunar de Tuxpan, Tampamachoco, Veracruz, México. Biótica 8 (2):201-213

De la Cruz-Francisco, V. 2012. Rhizophora mangle Linnaeus, 1753 como especie sombrilla y razón biológica para la protección y restauración de la laguna de Tampamachoco, Veracruz, México. BIOCYT 5 (18):341-352.

De la Lanza-Espino, G.; Sánchez-Santillán, N.; Esquivel-Herrera, A. 1998. Análisis temporal y espacial fisicoquímico de una laguna tropical a través del análisis multivariado. Hidrobiológica 8 (2):89-96.

Díaz, Y.J.; Guerra-García, J.M.; Martín, A. 2005. Caprellids (Crustacea: Amphipoda: Caprellidae) from shallow waters of the Caribbean coast of Venezuela. Organisms Diversity y Evolution 10:1-25.

García-Guerrero, M.; Hendrickx, M.E. 2003. Distribution of isopods (Peracarida, Isopoda) associated with prop roots of Rhizophora mangle in a tropical coastal lagoon, southeastern Gulf of California, México. Crustaceana 76:1153-1169.

García P, J.M.; Palacio, J. 2008. Macroinvertebrados asociados a las raíces sumergidas del mangle rojo (Rhizophora mangle) en las bahías Turbo y El Uno, Golfo de Urabá (Caribe Colombiano). Gestión y Ambiente 11 (3):55-66.

García-Cubas, A.; Reguero, M. 2004. Catálogo ilustrado de moluscos gasterópodos del Golfo de México y mar Caribe. Universidad Nacional Autónoma de México. México.

García-Cubas, A.; Reguero, M. 2007. Catálogo ilustrado de moluscos Bivalvos del Golfo de México y mar Caribe. Universidad Nacional Autónoma de México. México.

González, D.E.; Tzel, P.R.; Oyosa, O.G. 2008. Macroalgas del sistema de raíz de mangle. Jaina 19 (1):54-61.

Guerra-Castro, E.; Cruz-Motta, J.J.; Conde, J.E. 2011. Cuantificación de la diversidad de especies incrustantes asociadas a las raíces de Rhizophora mangle L. en el parque nacional laguna de la Restinga. Interciencia 36 (12):923-930. 
Heard, R. W. 1982. Guide to CommonTidal MarshInvertebrates of the Northeastern Gulf of México. Copyright. Mississippi.

Inclán-Rivadeneyra, I. 1989. Ecology of the epibiosis on the submerged roots of Rhizophora mangle in Bahía de laAscensión, Quintana Roo, México. Ciencias Marinas 15:1-20.

Jiménez-Valverde, A.; Hortal, J. 2003. Las curvas de acumulación de especies y la necesidad de evaluar la calidad de los inventarios biológicos. Revista Ibérica de Aracnología 8:151-161.

Lara Domínguez, A.L.; Contreras Espinosa, F.; Castañeda-López, O.; Barba-Macías, E.; Pérez-Hernández, M.A. 2011. Lagunas y estuarios. Pags. 297-313. En: Comisión Nacional para el conocimiento y Uso de la Biodiversidad (Conabio). La biodiversidad en Veracruz: Estudio de Estado. Comisión Nacional para el conocimiento y Uso de la Biodiversidad, Gobierno del Estado de Veracruz, Universidad Veracruzana, Instituto de Ecología, A. C. México.

Littler, D.S.; Littler, M.M. 2000. Caribbean ReefPlants. An Identifiation Guide to the ReefPlants of the Caribbean, Bahamas, Florida and Gulf of México. Offshore Graphics. Washington.

López-Ortega, M.; Pulido-Flores, G.; Serrano-Solís, A.; Gaytán-Ayarzún, J.C.; Monks-Sheets, W.S.; López-Jiménez, M.A. 2012. Evaluación estacional de las variables fisicoquímicas del agua de la Laguna de Tampamachoco, Veracruz, México. UDO Agrícola 12 (3):713-719.

López-Portillo, J.; Ezcurra, E. 2002. Los manglares de México: una revisión. Madera y Bosques 8:27-51.

López, B.; Vera, B.; Conde, J.E. 2009. Macroalgas asociadas a las raíces de Rhizophora mangle L. en la península de Paraguaná, Estado Falcón, Venezuela. Boletín del Instituto Oceanográfico de Venezuela 48 (1):11-21.

Márquez, B.; Jiménez, M. 2002. Moluscos asociados a las raíces sumergidas del mangle rojo Rhizophora mangle, en el Golfo de Santa Fe, Estado Sucre, Venezuela. Revista de Biología Tropical 50 (3/4):1101-1112.

Márquez-Rojas, B.; Blanco-Rambla, J.P.; Jiménez, M.; Allen, T. 2006. Crustáceos asociados a las raíces del mangle rojo Rhizophora mangle (L.) en el Golfo de Santa Fe, Estado Sucre, Venezuela. Ciencia 14 (1):12-27.

Medina, P.M., Mildred, E.M.; Polo, C.A.; Reyes, J.L.; Godoy, A.R. 2005. Isópodos en raíces de mangle rojo (Rhizophora mangle), en Isla San Carlos, Estado Zulia, Venezuela. Boletín del Centro de Investigaciones Biológicas 39 (1):67-79

Mills, C.E.; Marques, A.C.; Migotto, A.E.; Calder, D.R.; Hand, C. 2007. Hydrozoa: polyps, hydromedusae, and siphonophora. Págs. 118-167. En: Carlton, J.T. (Ed.). The Light and Smith manual: intertidal invertebrates from central California to Oregon. University of California Press, Berkeley.

Montes de Oca, S.P. 2010. Blenniidae, cachuditos, borrachos. Revista de Biología Tropical 58 (2):160-161.

Montes-M., A.; Prieto-Arcas, A.; Ruiz, L.J. 2007. Abundancia, biomasa y proporción sexual de una población natural de la ostra (Crassostrea rhizophorae) en la laguna Grande de Obispo, Estado Sucre, Venezuela. Boletín del Centro de Investigaciones Biológicas 41 (4):485-501.

Moreira G, A.R.; Gómez B, M.; León P,A.R.; del Pozo, P.; Cabrera G, R.; SuárezA, A.M. 2003. Variación de la composición y abundancia de macroalgas en el área protegida laguna Guanaroca, provincia de Cienfuegos, Cuba. Revista Investigaciones Marinas 24 (3):177-184.

Moreno, C.E.; Barragán, F.; Pineda, E.; Pavón, N.P. 2011. Reanálisis de la diversidad alfa: alternativas para interpretar y comparar información sobre comunidades ecológicas. Rev. Mex. Biodiv. 82:1249-1261

Naylor, E.E. 1972. British marine isopods. Keys and notes for the identification of the species. The linnean society of London. New York.

Ocaña, L. A.; Sánchez, R.M. 2003. Diversity of Ichthyoplankton in Tampamachoco lagoon, Veracruz, México. Anales del Instituto de Biología, Serie Zoología 74 (2): 179-193. 
Orihuela, B.; Díaz, H. 1991. Mass mortality in a mangrove roots fouling community in a hypersaline tropical lagoon. Biotropica 23 (4b):592-601.

Pérez-Mozqueda, L.L.; Del Castillo-Falconi, V.; Bortolini-Rosales, J.L. 2014. Registros adicionales del género Uca (Brachyura: Ocypodidae) en la laguna de Tamiahua, Veracruz, México. Rev. Mex. Biodiv. 85 (3):969-971.

Pizarro, P.; Piedra, L.; Bravo, J.; Asch, J.; Asch, C. 2004. Manual de procedimiento para el manejo de los manglares de. Costa Rica. EFUNA.

Prüsmann, J.; Palacio, J. 2008. Colonización de moluscos y crustáceos en raíces de mangle rojo en la laguna costera de la punta norte del golfo de Morrosquillo. Gestión y Ambiente 11 (3):77-86.

Quiceno C, P.A.; Palacio B, J.A. 2008. Aporte al conocimiento de los macroinvertebrados asociados a las raíces del mangle (Rhizophora mangle) en la ciénega la Boquilla, municipio de San Onofre, Sucre. Gestión y Ambiente 11 (3):67-78.

Quirós-Rodríguez, J.A.; Arias, J.E. 2013. Taxocenosis de moluscos y crustáceos en raíces de Rhizophora mangle (Rhizophoraceae) en la bahía de Cispatá, Córdoba, Colombia. Acta Biológica Colombiana 18 (2):329-340.

Quirós-Rodríguez, J.A.; Medrano-Mangones, W.J.; Santafé-Patiño, G.G. 2017. Esponjas (Porifera: Demospongiae) de raíces sumergidas de Rhizophora mangle en la bahía de Cispatá, Córdoba, Caribe Colombiano. Revista Mexicana de Biodiversidad 88 (1):80-85.

Reyes, R.; Campos, N.H. 1992. Macroinvertebrados colonizadores de raíces de Rhizophora mangle en la bahía de Chengue, Caribe Colombiano. Anales del Instituto de Investigaciones Marinas Punta Betín 21:101-116.

Romero-Murillo, P.E.; Polanía, J. 2008. Sucesión temprana de la taxocenosis Mollusca-Annelida-Crustacea en raíces sumergidas de mangle rojo en San Andrés Isla, Caribe colombiano. Revista de Biología Marina y Oceanografía 43 (1):63-74.

Ruiz G, M.R.; López-Portillo, J. 2017. Colonización y supervivencia de epibiontes sésiles en substratos artificiales similares a rizóforos de Rhizophora mangle (Rhizophoraceae) en La Mancha, México. Revista de Biología Tropical 65 (2):745-761.

Ruiz, M.; López-Portillo, J. 2014. Variación espacio-temporal de la comunidad de macroinvertebrados epibiontes en las raíces del mangle rojo Rhizophora mangle (Rhizophoraceae) en la laguna costera de La Mancha, Veracruz, México. Revista de Biología Tropical 62 (4):1309-1330.

Salgado B, J.; Hendrickx, M.E. 2002a. Distribution and abundance of bernacles (Crustacea: Cirripedia) associated with prop roots of Rhizophora mangle L. in a lagoon of Northwest Mexico. Págs. 45-60. En: Hendrickx (Ed.) Contributions to the study of East Pacific Crustaceans. Instituto de Ciencias del Mar y Limnología, UNAM. México.

Salgado B, J.; Hendrickx, M.E. 2002b. Panopeid crabs (Crustacea:Brachyura: Panopeidae) associated with prop roots of Rhizophorae mangle L. in a tropical coastal lagoon of the SE Gulf of California, México. Págs. 1-7. En: Hendrickx (Ed.) Contributions to the study of East Pacific Crustaceans. Instituto de Ciencias del Mar y Limnología, UNAM. México.

STATSOFT. 2005. Statistica (data analysis software system), version 7.1.EE.UU.: Tulsa, OK, StatSoft, Inc.

Vilardy, S.; Polanía, J. 2002. Mollusc fauna mangrove root-fouling community at the Colombian Archipelago of San Andres and Old Providence. Wetlands Ecology and Management 10:273-282.

Vilchis A., M. I. 2015. Biogeografía de las grandes macroalgas en el golfo de México y Caribe Mexicano. Tesis de maestría. Universidad Autónoma Metropolitana.

Walther, B.A.; Moore, J.L. 2005. The concepts of bias, precision and accuracy, and their use in testing the performance of species richness estimator, with a literature review of estimator performance. Ecography 28:815-829.

Weerdt, W.H.; Rützler, K.; Smith, K.P. 1991. The Chalinidae (Porifera) of twincays, Belize, and adjacent waters. Proc. Biol. Soc. Wash. 104 (1):189-205. 